plan. On joining the health plan in the 1960s and 1970s before diagnosis of their MS, the patients had answered a health questionnaire, undergone multiphasic examinations, and donated blood specimens for long-term storage as frozen serum. For each patient, three healthplan members without MS acted as matched controls. Analysis of the serum samples was carried out to determine levels of antibodies against the Epstein-Barr nuclear antigen (EBNA) complex, and the date of symptom onset for patients with MS was reviewed.

The geometric mean antibody titers to the EBNA complex and one of its components, EBNA-1, were significantly higher in patients with $\mathrm{MS}$ than in controls $(P=0.007$ for EBNA and $P=0.01$ for EBNA-1). Moreover, these elevations were found to be present 15-20 years before the onset of MS neurological symptoms, and remained constant over time.

The authors conclude that elevated antiEBV levels are probably part of the early pathogenesis of MS. They highlight the importance of determining the mechanisms connecting EBV and MS, to aid future research into MS treatment and prevention.

Original article DeLorenze GN et al. (2005) Epstein-Barr virus and multiple sclerosis: evidence of association from a prospective study with long-term follow-up. Arch Neurol [doi: 10.1001/archneur.63.6.noc50328]

\section{New tool to alert clinicians to nonmotor symptoms in Parkinson's disease}

Despite causing considerable disability, nonmotor symptoms (NMS) of Parkinson's disease (PD), such as depression, sleep problems, pain, apathy, balance trouble and memory problems, are under-recognized and undertreated. A multidisciplinary group of experts from around the world has developed a 30-item screening questionnaire (NMSQuest) for assessing NMS in patients with PD before they are seen in the clinic, to alert clinicians to problems that might require further investigation.

Completed NMSQuest data from 123 patients with PD were analyzed, and responses compared with those from 96 healthy, agematched controls. NMS were much more common in patients with PD than in controls. Among others, complaints of dribbling, impaired taste or smell, impaired swallowing, constipation, urinary urgency, sadness, concentration difficulties, anxiety and dizziness were significantly more common in PD patients than in controls $(P<0.05$ for all). The median number of NMS was nine in patients with PD compared with four in the control group. The total number of NMS present increased both with PD severity and with disease duration. In a survey of a subset of 14 patients and 13 caregivers, $90 \%$ thought that the items contained in NMSQuest were relevant, and over $75 \%$ stated that NMSQuest improved doctor treatment of PD.

The authors conclude that a wide range of NMS, many of which are not usually reported to clinicians, occur across all stages of PD. They recommend use of NMSQuest as a screening tool while patients are waiting to be seen by clinicians, to identify problems and improve patient treatment.

Original article Chaudhuri KR et al. (2006) International multicenter pilot study of the first comprehensive self-completed nonmotor symptoms questionnaire for Parkinson's disease: the NMSQuest study. Mov Disord [doi: 10.1002/mds.20844]

\section{Strong support for partial integration of neurology and psychiatry teaching}

A recent survey conducted in the UK investigated the attitudes of doctors within neurology and psychiatry specialties to the concept of closer educational links between the two disciplines; the results indicate strong, widespread support for such a transition.

Schon and colleagues sent questionnaires to the undergraduate coordinators of neurology and psychiatry teaching in all 26 UK medical schools, all 31 members of the specialist advisory committees of the Royal College of Physicians in Neurology and the Royal College of Psychiatrists in General and Old Age Psychiatry, and 40 postgraduate trainees in each discipline. Their questionnaire was composed of two main sections: the first examined general attitudes to links between the disciplines, and the second presented a list of common topics that could be suitable for integrated teaching, and asked respondents to comment on the degree to which they thought the topic would be suitable.

The overall response rate to the questionnaire was over $60 \%$. More than $75 \%$ of respondents had a personal inclination for closer links between the disciplines, with psychiatrists 